\title{
Prescribing pattern of antibiotics in the department of pediatrics in a tertiary care medical college hospital in Northern India
}

\author{
Ravika Kanish', Kanchan Gupta², Shivani Juneja², H.S. Bains ${ }^{3}$, Sandeep Kaushal ${ }^{2}$ \\ ${ }^{1}$ Final Year MBBS Student, ${ }^{2}$ Department of Pharmacology, ${ }^{3}$ Department of Pediatrics, Dayanand Medical College and Hospital, Ludhiana, Punjab
}

\section{A B S T R A C T}

Background: Systemic antibiotics account for more than one-third of all prescriptions in children; hence, antibiotic prescriptions in children are a major public health concern. Moreover, data regarding rational antibiotic use in children is very limited. Hence, it is essential that the antibiotic prescribing pattern be evaluated periodically for its rationality of use and cost. The aim of our study is to identify the prescribing pattern and to carry out direct cost analysis of antibiotic use in the patients admitted in pediatrics department of a tertiary care hospital of North India. Methods: The study was conducted for two months (June, July, 2012) in pediatric ward and ICU. The data regarding patient demographics and antibiotic use was collected daily in a structured proforma. A descriptive analysis of the data was done. Results: A total of one hundred and ninety one patients were enrolled, most of them belonging to the age group 2-14 years. Majority of them were male pediatric patients $(75.9 \%)$ with mean age of 4.6 years. On an average 1.9 AMAs were prescribed per patient. The most common AMAs prescribed were cephalosporins followed by aminoglycosides. The preferred route of administration was injectable (92\%). The average direct cost of treatment per patient was estimated to be Rs. 3338. The percentage of drugs prescribed by generic name was $58 \%$. Conclusions: Male pediatric patients have better access to tertiary medical care as compared to female pediatric patients. The average number of anti-microbials prescribed is 1.9 which is not too high. Newer generation of antibiotics are more commonly prescribed leading to increased cost of therapy. This baseline study will help in formulating an antibiotic policy and standard treatment guidelines for appropriate use of antibiotics.

Key words: Antibiotics, antibiotic resistance, pediatrics, pharmaco-economics, prescribing pattern

\section{INTRODUCTION}

Drug utilization study (DUS) is the study of marketing, distribution, prescription, and use of drugs in a society, with special emphasis on the resulting medical, social and economic consequences. ${ }^{1}$ The pattern of drug utilization may be linked to the reasons of drug prescribing. ${ }^{2}$ Also, controlling cost is a vital issue, since, in developing countries like India, compliance is primarily dependent on the cost of treatment. Therefore, it has implications not only on the medical but also on social and economic aspects. ${ }^{3}$ The indiscriminate use of antibiotics often results in increased
Access this article online

Website:

http://nepjol.info/index.php/AJMS 
trends in clinical settings and the associated adverse drug reactions which will serve as a baseline data for formulating standard treatment guidelines to promote rational antibiotic use. Evaluation of prescribing pattern will also help in minimizing adverse drug reactions as children are more susceptible to them and it shall also aid in providing cost effective medical care. ${ }^{8,9}$ Therefore, this study would help us to monitor the prescribing pattern and cost of antibiotics so that these can be used judiciously.

\section{METHODS}

\section{Study design}

This was a hospital based prospective and observational study carried out over a period of two months (June and July, 2012).

\section{Study population}

The study was done in the Department of Pediatrics of a tertiary care hospital in Northern India. The data was collected from patients admitted to pediatric ward and ICU over these two months. The hospital caters to both urban and rural population. Most of the patients belong to middle and upper strata of the society.

\section{Sampling method}

All the patients up to the age of 18 years who were on antibiotic prescription and were willing to give consent were included in the study. The pediatric ward and ICU were visited on all seven days of the week and information regarding the patient demographics and antibiotic use were recorded in a semi-structured proforma. A total of 225 patients were screened out of which 26 patients were not prescribed any antibiotic and 8 patients refused to give consent. These patients were excluded from the study. The remaining 191 patients were included in the study.

\section{Ethical approval}

The study was approved by the Institutional Ethics Committee. The consent forms in all the three languages prevalent in the region (Punjabi, Hindi and English) were made available and consent of each patient/guardian was taken.

\section{Data analysis}

The prescriptions were analyzed for the average number of antibiotics per encounter, percentage of encounters with an antibiotic prescribed, route of administration based prescription pattern, group wise prescription of antibiotics, percentage of antibiotics prescribed by generic name, percentage of antibiotics prescribed from the WHO essential drug list, outcome and cost analysis. The data was pooled and descriptive analysis done. The results were presented as mean and percentages.

\section{RESULTS}

A total of 191 pediatric patients fulfilled the inclusion criteria and were enrolled. Out of them, $145(75.9 \%)$ were male and $46(24.1 \%)$ were female. The age wise distribution of patients is depicted in Table 1 .

The timing of delivery with respect to gestational period was also studied and $169(88 \%)$ deliveries were conducted at term gestation, $10(5.23 \%)$ deliveries were preterm and $12(6.28 \%)$ deliveries were conducted at post term gestation.

Average no. of antibiotics received per patient was 1.9. Percentage of encounters with an antibiotic was $91.6 \%$. Parenteral drugs constituted $92 \%$ of the total number of AMAs prescribed. On analyzing the route of administration of drugs 330 (92.21\%) were given parenterally, $24(6.70 \%)$ were given through oral route, $2(0.55 \%)$ were given intramuscularly and $1(0.27 \%)$ each was given intraocularly and topically.

The major complaints of the patients were fever and other symptoms related to respiratory and GI system for which they were prescribed antibiotics. The most common antibiotics prescribed were cephalosporins $(39 \%)$ followed by aminoglycosides (23\%), fluoroquinolones (11\%), and beta lactams (9.9\%) (Excluding cephalosporins), miscellaneous $(7.9 \%)$, anti-anaerobic (metronidazole) $(4.8 \%)$ and macrolides $(4.8 \%)$. The pattern of antibiotic prescription is depicted in Figure 1.

The subgroup analysis of prescription of cephalosporins is shown in Figure 2.

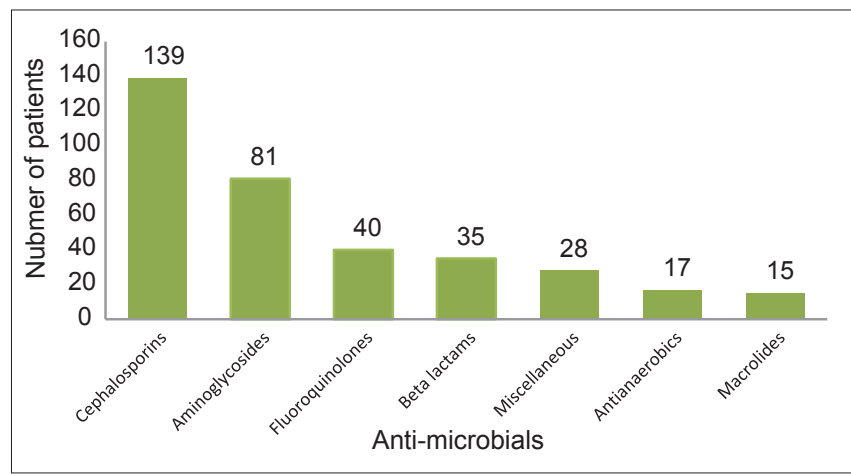

Figure 1: Pattern of prescription of anti-microbials

Table 1: Agewise distribution of patients $(n=121)$

\begin{tabular}{lcc}
\hline Age group & No. of patients (\%) & Mean age \pm SD \\
\hline$<12$ months & $67(35.07)$ & $5.18 \pm 3.55$ months \\
$12-24$ months & $22(11.51)$ & $14.81 \pm 2.87$ months \\
$2-14$ years & $100(52.35)$ & $7.61 \pm 3.74$ years \\
$14-18$ years & $2(1.04)$ & $15 \pm 0.35$ years \\
\hline
\end{tabular}




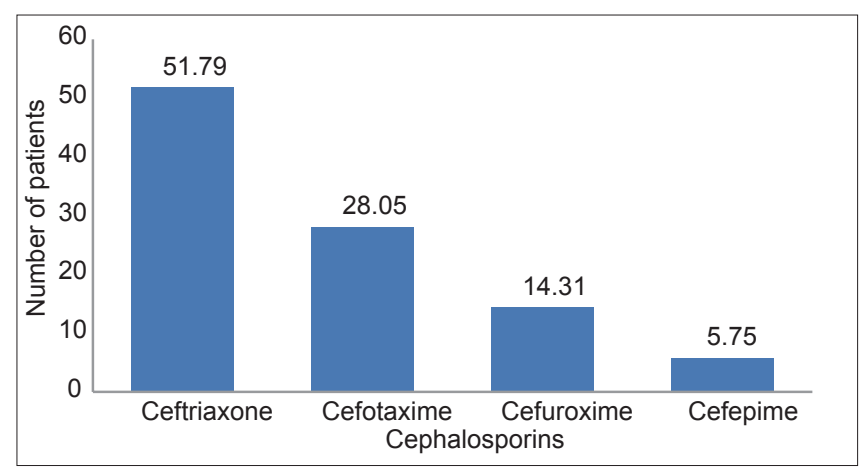

Figure 2 : Spectrum of cephalosporin prescription

Amikacin (87.7\%) among aminoglycosides; amoxicillin plus clavulanic acid $(20 \%)$ among beta lactams (except cephalosporins) and ofloxacin (52.5\%) among fluoroquinolones were prescribed most commonly.

The average duration of stay of patients was found to be 10.8 days. The overall rate of single drug prescription was $17 \%$ and that of fixed drug combinations was $4 \%$. And out of the total number of AMAs prescribed, generic prescription rate was 58\%. Overall, $86 \%$ of the total drugs were from the WHO List of Essential Medicine, 2010.

A favourable outcome was seen in $84.7 \%$ of the patients. Average expenditure of the AMAs per patient for the total hospital stay was Rs. 3338. The cost incurred on different group of antibiotics is depicted in Figure 3.

\section{DISCUSSION}

The present study provides us with an overall pattern of antibiotic use profile in patients admitted in pediatric ward and ICU in a tertiary care hospital. Majority of the patients were males as was also seen in another study. ${ }^{10}$ This can be attributed to the prevalence of gender inequality as a result of which males are preferentially taken to tertiary care institutes for treatment as compared to females with a similar severity of illness. ${ }^{11}$ Another observation was the greater number of full term deliveries, hence pointing towards the better antenatal care of the patients catered by our hospital.

Majority of the patients $(91.6 \%)$ were prescribed at least one antibiotic which is many times more than what was found in another study. ${ }^{12}$ The average number of antibiotics being prescribed per patient is 1.9, which is less than that observed in a similar study conducted in pediatric patients. ${ }^{13}$

As already mentioned, total antibiotic usage included both intravenous and oral antibiotics, of which beta-lactams

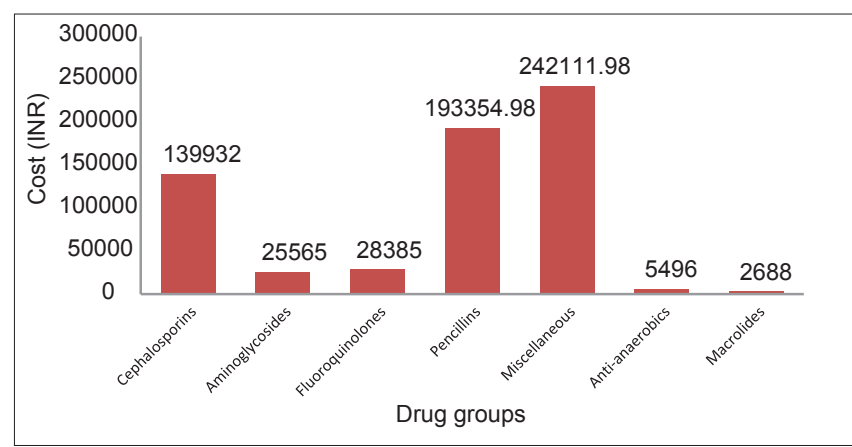

Figure 3: Cost wise antibiotic prescription

(cephalosporins the most) were maximally prescribed followed by aminoglycosides which is also the trend seen in another study. ${ }^{14}$ Among the beta-lactams, cephalosporins were prescribed maximally, which is similar to another study. ${ }^{13}$ Third generation cephalosporins were prescribed more frequently, which may be because ours is a tertiary care hospital and patients would have already been administered and probably developed resistance to lower generation antibiotics like co-trimoxazole, chloramphenicol, penicillinase resistant penicillins (methicillin, oxacillin, cloxacillin) etc., Culture sensitivity was done in most of the patients but due to prior exposure to antibiotics (before reaching the hospital), the cultures are negative most of the times.

Majority of the drugs were given by intravenous route followed by the oral route as also identified in a study conducted on antibiotic use in pediatrics. ${ }^{15}$ This mainly indicates that patients in wards or ICUs necessarily require the IV route for urgent control of infections and to minimize morbidity as compared to oral route.

Although according to our study, there were a fairly good percentage of prescriptions by generic names but there is still a long way to go before we thoroughly inculcate this habit which will again help in reducing the cost, as was also concluded by another study. ${ }^{16}$ Thus, more information about generic drugs and more research in the field of pediatric pharmacology are needed for increasing generic medicine prescription rate. ${ }^{17}$

The average cost incurred per patient was Rs 3338. Beta lactams followed by aminoglycosides accounted for the maximal cost of the antimicrobials. Miscellaneous antibiotics which included Vancomycin, Linezolid, Tigecycline, Bacitracin and Doxycycline also contributed to a significant extent to the total cost of AMAs (Figure 3). Patients reach tertiary care hospital (like ours) in advanced stage with prior appropriate/inappropriate exposure to antibiotics and it becomes an absolute necessity to use higher generations of antibiotics. This leads to increased 
cost of therapy. The antibiotics are gradually de-escalated based upon the patient response and protocol of treatment.

Moreover, due to feasibility purpose, we are mentioning only the direct cost of the antibiotics, whereas there are various indirect expenses involved in a patient's hospital stay which cost the patient many times more.

To conclude, male pediatric patients have better access to tertiary medical care as compared to female pediatric patients. The average number of anti-microbials prescribed is 1.9 which is not too high. Newer generation of antibiotics are more commonly prescribed leading to increased cost of therapy.

Antibiotics are the most commonly prescribed drugs in pediatric population, thus special measures are imperative for their rational usage to prevent emergence of resistance. ${ }^{14,18}$ This preliminary study has given an insight into the pattern of AMA prescribing upon which future intervention studies may be based to promote rational drug use. Antibiotic policy and standard treatment guidelines are being framed.

Although our study has been conducted using a reasonably good sample size making various parameters quite trustworthy but the limitation of our study could be the smaller sample size. The robustness of our findings could have been increased by an even larger sample size had the duration of study been longer. Another limitation is that it is a single centre study, hence the validity of findings would increase if it is a multi-centre study.

As number of pediatric drug utilization studies is very small, we must seek ways and means to rationalize and prioritize which antibiotics must be prescribed in pediatric patients who would maximally benefit from them as was the aim of another study. ${ }^{19}$ This would be best articulated through preparation and circulation of standard treatment guidelines (STG) which is the ultimate goal of our study.

\section{REFERENCES}

1. Introduction to Drug Utilisation Research. What is drug utilisation research and why it is needed? Available from http:Ilapps. who.intlmedicinedocslenldlJs4876el 2.1.html. Accessed on November 16 $6^{\text {th }}, 2011$.

2. Gama H. Drug Utilisation Studies. Arq Bras Med 2008; 22:69-74.

3. Zuppa A, Vijaykumar S, Jayaraman B, Patel D, Narayan M, Vijaykumar K, et al. An informatics approach to assess paediatric pharmacotherapy: Design and implementation of a hospital drug utilisation system. J Clin Pharmacol 2007; 47:1172-1180.

4. Thrane N, Olesen C, Schonheyder HC and Sorensen HT. Socioeconomic factors and prescription of antibiotics in 0- to 2-year-old Danish children. J Antimicrob Chemother 2003:51: 683-689.

5. Majeed A and Moser K. Age- and sex-specific antibiotic prescribing patterns in general practice in England and Wales in 1996. Br J Gen Pract 1999; 49:735-736.

6. Grohskopf LA, Huskins WC, Sinkowitz-Cochran RL, Levine GL, Goldmann DA and Jarvis WR. Paediatric Prevention Network Use of antimicrobial agents in United States neonatal and paediatric intensive care patients. Pediatr Infect Dis J 2005; 24:766-773.

7. Katragkou A, Kotsiou $M$ and Antachopoulos C. Acquisition of imipenem-resistant Acinetobacter baumannii in a paediatric intensive care unit: a case control study. Intens Care Med 2006; 32:1384-1391.

8. Introduction to drug utilisation research. WHO International Working Group for Drug Statistics Methodology, WHO Collaborating Center for Drug Statistics Methodology, WHO Collaborating Center for Drug Utilisation Research and Clinical Pharmacological Services. Geneva: WHO; 2003.

9. Tabatabaei SA, Fahimzad SA, Shamshiri AR, Shiva F, Salehpor S, Sayyahfar S, et al. Assessment of a new algorithm in the management of acute respiratory tract infections in children. J Res Med Sci 2012; 17:182-185.

10. De Bont EG, van Loo IH, Dukers-Muijrers NH, Hoebe CJ, Bruggeman CA, Dinant GJ, et al. Oral and topical antibiotic prescriptions for children in general practice. Arch Dis Child 2012; [Epub ahead of print].

11. Palikhe N. Prescribing Pattern of Antibiotics in Paediatric Hospital of Kathmandu Valley. Kathmandu Univ Med J 2004; 2:6-12.

12. Alvarez-Lerma F. Modification of antibiotic treatment in patients with pneumonia acquired in the intensive care unit. ICU-Acquired Pneumonia Study Group. Intensive Care med 1996; 22:387-394.

13. Van Houten MA, Luinge K, Laseur M and Kimpen JL. Antibiotic utilisation for hospitalised paediatric patients. Int J Antimicrob Agents 1998; 10:161-164.

14. Pandey AA, Thakre SB and Bhatkule PR. Prescription analysis of pediatric outpatient practice in Nagpur city. Indian J Community Med 2010; 35:70-73.

15. Dimri S, Tiwari P, Basu S and Parmar VR. Drug use pattern in children at a teaching hospital. Indian Pediatr 2009; 46:165-167.

16. Shrestha R, Shrestha JM and Gurung B. Antibiotic Usage and its Sensitivity Pattern in the NICU. Kathmandu Univ Med J 2012; 10:27-32.

17. Altiner A, Berner R, Diener A, Feldmeier G, Kochling A, Loffler C, et al. Converting habits of antibiotic prescribing for respiratory tract infections in German primary care-the cluster-randomized controlled CHANGE-2 trial. BMC Fam Pract 2012; 13:124.

18. Shrestha R, Shrestha JM and Gurung B. Antibiotic Usage and its Sensitivity Pattern in the NICU. Kathmandu Univ Med J 2012; 10:27-32.

19. Altiner A, Berner R, Diener A, Feldmeier G, Kochling A, Loffler C, et al. Converting habits of antibiotic prescribing for respiratory tract infections in German primary care-the cluster-randomized controlled CHANGE-2 trial. BMC Fam Pract 2012; 13:124.

\section{Authors Contribution:}

RK - Data collection and analysis, drafting the manuscript; KG - Conception and study design, data interpretation, drafting the manuscript; SJ - Data collection and analysis, drafting the manuscript; SK - Data interpretation, reviewed the manuscript; HSB - Reviewed the manuscript. 